\title{
RETROSPECTIVA DEL BOSQUE DE PINO Y ENCINO DE LA SIERRA MADRE OCCIDENTAL, SONORA, NOROESTE DE MÉXICO, HACE 1000 AÑOS
}

\author{
Carmen Isela Ortega-Rosas ${ }^{1, *}$, M. Cristina Peñalba ${ }^{1, * *}$, José Antonio López-Sáez ${ }^{2}$, \\ Thomas R. VAn DeVEndeR ${ }^{3}$
}

${ }^{1}$ Universidad Nacional Autónoma de México, Instituto de Ecología, Apdo. postal 1354, 83000 Hermosillo, Sonora, México.

${ }^{2}$ Consejo Superior de Investigaciones Científicas, Instituto de Historia, Departamento de Prehistoria, Laboratorio de Arqueobotánica, Duque de Medinaceli 6, 28014 Madrid, España. alopez@ceh.csic.es

${ }^{3}$ Arizona-Sonora Desert Museum, 2021 N. Kinney Rd., Tucson, AZ 85743, Estados Unidos.yecora4@comcast.net

\section{RESUMEN}

La Ciénega de Camilo es un humedal con Sphagnum palustre localizado en un bosque denso de pino y encino con cuatro especies de Pinus y siete de Quercus, en la Sierra Madre Occidental, en la región este de Sonora. Los análisis de polen y palinomorfos no polínicos de dos núcleos de sedimento muestran que el bosque de pino y encino ha existido en el lugar durante el último milenio. Sin embargo, hace alrededor de mil años (1058 \pm 60 y $870 \pm 70$ años cal. BP (años calibrados antes del presente)), el pino era más abundante que hoy día, y además crecía una especie de pino adicional, posiblemente del grupo de Pinus strobiformis, que hoy prospera a mayores elevaciones, en un clima más fresco y húmedo. Había abundancia y diversidad de helechos. Los palinomorfos no polínicos sugieren condiciones más húmedas (presencia de Copepoda) y eu- a mesotróficas en la base, seguidas por condiciones más secas (amerosporas indiferenciadas, tipo 55A, Zygnemataceae y Pediastrum), conducentes al medio ambiente mesotrófico-ombrotrófico más reciente caracterizado por Pleospora y tipo 82E, que refleja la ciénega de Sphagnum palustre. Se infiere que hubo

\footnotetext{
* Dirección actual: Centre Européen de Recherche et d'Enseignement des Géosciences de l'Environnement, BP 80, Europôle Méditerranéen de l'Arbois, 13545 Aix-en-Provence cedex 4, Francia. ortega@cerege.fr

${ }^{* *}$ Dirección actual: Universidad de Sonora, Departamento de Geología, Apdo. postal 847, 83000 Hermosillo, Sonora, México. penalba@servidor.unam.mx
} 
un período húmedo hace alrededor de 1000 años $\left({ }^{14} \mathrm{C} \mathrm{BP}\right)$, contemporáneo con el máximo desarrollo de las culturas arqueológicas de los desiertos Sonorense y Chihuahuense, que fue seguido por un clima progresivamente más seco y cálido.

Palabras clave: cambio climático, Holoceno reciente, México, paleoecología, palinomorfos no polínicos, polen.

\section{ABSTRACT}

The Ciénega de Camilo is a Sphagnum palustre seep in a canyon in dense pine-oak forest with four species of pine and seven of oak in the Sierra Madre Occidental of eastern Sonora, Mexico. Analyses of pollen and non-pollen palynomorphs in two sediment cores show that pine-oak forest has been at the site during the last thousand years. When sedimentation began about 1000 years ago (1058 \pm 60 and $870 \pm 70$ cal. BP (calibrated years before present)), pine was more abundant than today with an additional species with large pollen grains now present at higher elevation. The abundance and diversity of ferns were higher. Nonpollen palynomorphs suggest moister (presence of Copepoda), and eu- to mesotrophic conditions at the base, followed by drier conditions (unidentified amerospores, type 55A, Zygnemataceae, and Pediastrum), leading to the most recent mesotrophic-ombrotrophic environment characterized by Pleospora, type 82E, and the Sphagnum palustre seep. The inferred wet period at around 1000 cal. BP followed by drier and warmer climates is contemporaneous with the maximum development of the Sonoran and Chihuahuan desert archeological cultures.

Key words: climate change, Late Holocene, Mexico, non-pollen palynomorphs, palaeoecology, pollen.

\section{INTRODUCCIÓN}

El noroeste de México tiene una alta biodiversidad, reflejo de la transición entre los reinos Neotropical y Holártico, y de un fuerte gradiente altitudinal. Así, en el municipio de Yécora en el este de Sonora, por encima del matorral espinoso y la selva baja caducifolia, se suceden el pastizal templado, el bosque abierto de encino y el bosque de pino y encino. La flora, con cerca de 1700 taxa que crecen en $3300 \mathrm{~km}^{2}$, es muy diversa, con elementos de afinidad templada, tropical y con los desiertos Sonorense y Chihuahuense (Van Devender y Reina G., 2005; Van Devender et al., 2005). Sin embargo, poco se conoce del efecto que tuvieron los cambios climáticos pasados en la diversidad de plantas y en la historia de la vegetación del norte de la 
Sierra Madre. Trabajos previos no publicados (Ortega-Rosas, 2000, 2003) señalan la existencia de un registro de vegetación del Holoceno (los últimos 10,000 años) para la Ciénega de Camilo, en el municipio de Yécora. Presentamos aquí un estudio polínico de esta misma ciénega que muestra un registro paleoecológico que se remonta a hace mil años.

Algunos estudios previos aportan información sobre los paleoclimas del noroeste de México. Estos incluyen secuencias polínicas (Sirkin et al., 1994; Anderson y Van Devender, 1995; Ortega-Rosas, 2000, 2003; Lozano-García et al., 2002; Rhode, 2002), macrofósiles en depósitos de roedores (Van Devender et al., 1987; Van Devender, 1990a, 1990b), y secuencias de sedimento y diatomeas (Ortega-Ramírez et al., 1998; Ortega-Guerrero et al., 1999; Metcalfe et al., 2002), así como datos del suroeste de los Estados Unidos (Martin, 1963; Anderson y Shafer, 1991). Tales estudios sugieren que durante el Holoceno (últimos 11,000-10,000 años) la estacionalidad de las precipitaciones cambió drásticamente, ocasionando una mayor aridez y mayor calor al final de la primavera y en el inicio del verano, lo que culminó con el establecimiento del régimen climático moderno hace 4000 años, con el inicio del Holoceno reciente. Sin embargo, algunas investigaciones sugieren una aridez máxima en el Holoceno medio (Ortega-Ramírez et al., 1998; Ortega-Guerrero et al., 1999; Metcalfe et al., 2002), mientras para otros éste fue un período más húmedo (Martin, 1963; Van Devender et al., 1987; Van Devender, 1990a, 1990b; Ortega-Rosas, 2003).

También se registraron fluctuaciones de temperatura y humedad de menor magnitud en los últimos 2000 años, una de las cuales es un período más húmedo alrededor de 1000 BP (años de radiocarbono antes del presente, considerándose como presente el año 1950 de nuestra era) detectado por Van Devender (1987) y Van Devender et al. (1987). Estos cambios fueron concomitantes con el impacto humano (Sirkin et al., 1994; Sankey et al., 2001; Davis et al., 2002). Precisamente, este último período es de importancia clave en la historia de los habitantes pre-europeos de la región conocida como Aridamérica del norte de México. Existen evidencias arqueológicas que sugieren que hubo comercio con otras culturas después del año 1250 BP (1250 años antes del presente o año 700 de nuestra era según Álvarez-Palma, 1996). Sin embargo, alrededor de hace 1000 años, las interacciones ya fueron significativas. En Trincheras, en el centro-oeste de Sonora (Fig. 1), se practicaba la agricultura utilizando canales de irrigación entre los años 1150 y 850 BP, mientras que los primeros pueblos de la cultura del Río Sonora se registran en el año 875 BP. Por otra parte, en Casas Grandes en el noroeste de Chihuahua, hubo innovaciones considerables en las pautas de asentamiento, la arquitectura, decoración de cerá- 
Acta Botanica Mexicana 83: 69-92 (2008)

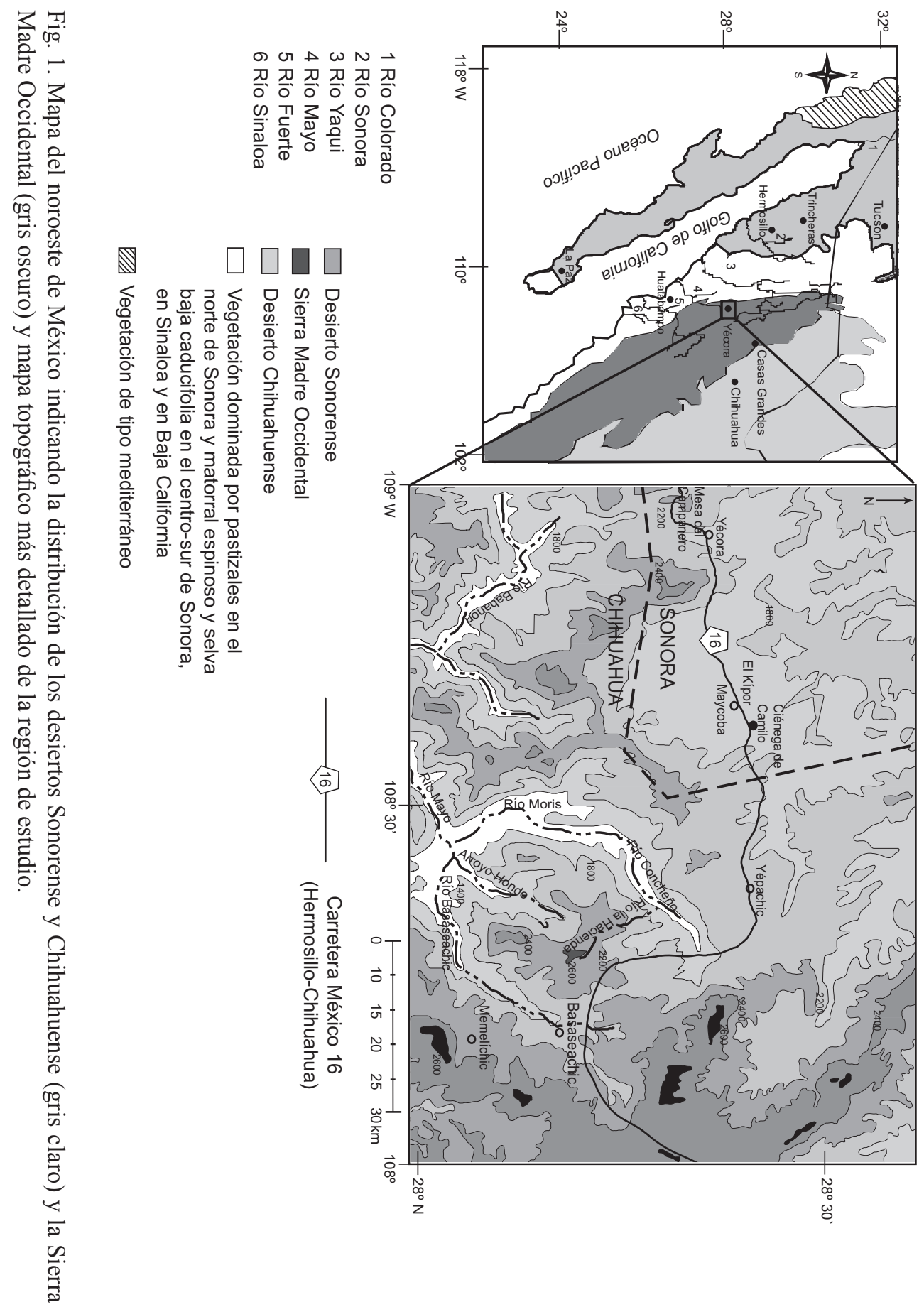


micas, etc., poco antes del año 890 BP (DiPeso, 1966; Álvarez-Palma, 1996). Los restos arqueológicos de la cultura Huatabampo de la costa sur de Sonora parecen confirmar este período húmedo: dichos asentamientos fueron abandonados hacia el año 950 BP debido a fuertes cambios en el lecho del río que causaron inundaciones en sus áreas de habitación. Los arqueólogos sugieren que hubo alteraciones en el régimen climático (Álvarez-Palma, 1996), pero no hay un registro paleoclimático disponible. Presentamos aquí un estudio de la historia de la vegetación y el clima en un sitio localizado en el bosque de pino y encino de la Sierra Madre Occidental, en el este de Sonora, a través del análisis del polen y los palinomorfos no polínicos (PNP) de una secuencia de sedimento fechada en $870 \pm 70$ y $1058 \pm 60$ cal. BP.

\section{ÁREA DE ESTUDIO}

La Sierra Madre Occidental es una de las principales provincias fisiográficas de México. Se trata de una gran formación de varios kilómetros de espesor, originada en el Oligoceno-Mioceno (Swanson y Walk, 1988; Cochemé y Demant, 1991; Roldán y Clark, 1992) que se desarrolla a lo largo de la costa occidental pacífica mexicana y separa los desiertos de Sonora y Chihuahua (Fig. 1). Las mayores elevaciones entre 27 y $30^{\circ}$ de latitud norte alcanzan $2700 \mathrm{~m}$. La precipitación anual en la localidad de Yécora, localizada a $1500 \mathrm{~m}$ de altitud en el este de Sonora, es en promedio de $913 \mathrm{~mm}$, siendo predominantes las lluvias de verano, la temperatura media anual es de $14.4^{\circ} \mathrm{C}$ (Búrquez et al., 1992). El sitio de estudio de la Ciénega de Camilo se localiza en el bosque de pino y encino entre Yécora y la frontera con el estado de Chihuahua (2825'34" N, 108 $34^{\prime} 09^{\prime \prime}$ W, 1550 m s.n.m.; Van Devender et al., 2003), $8.5 \mathrm{~km}$ al oeste de la frontera interestatal sobre la carretera 16 (Figs. 1 y 2). Es un área pantanosa cubierta de Sphagnum palustre (Fig. 3) de $1700 \mathrm{~m}^{2} \mathrm{de}$ superficie y $110 \mathrm{~m}$ de largo, ligeramente escalonada, con una pendiente media de $13^{\circ}$ (Ortega-Rosas, 2000), originada por una falla en el terreno, sobre rocas terciarias, y alimentada por una fuente permanente de agua ácida con pH 4.2 (Van Devender et al., 2003). Este hábitat es la única localización conocida de Sphagnum palustre en el noroeste de México. El sitio está rodeado por un bosque denso y diverso de pino y encino, con cuatro especies de pinos (Pinus engelmannii, P. yecorensis, P. chihuahuana, y $P$. herrerae) y siete de encinos (Quercus arizonica, $Q$. chihuahuensis, $Q$. coccolobifolia, $Q$. durifolia, $Q$. hypoleucoides, $Q$. oblongifolia y $Q$. toumeyi), y ocasionalmente con táscate (Juniperus deppeana) y madroño (Arbutus xalapensis) (Van Devender et al., 2003). En el sitio y a lo largo del arroyo que corre por el fon- 


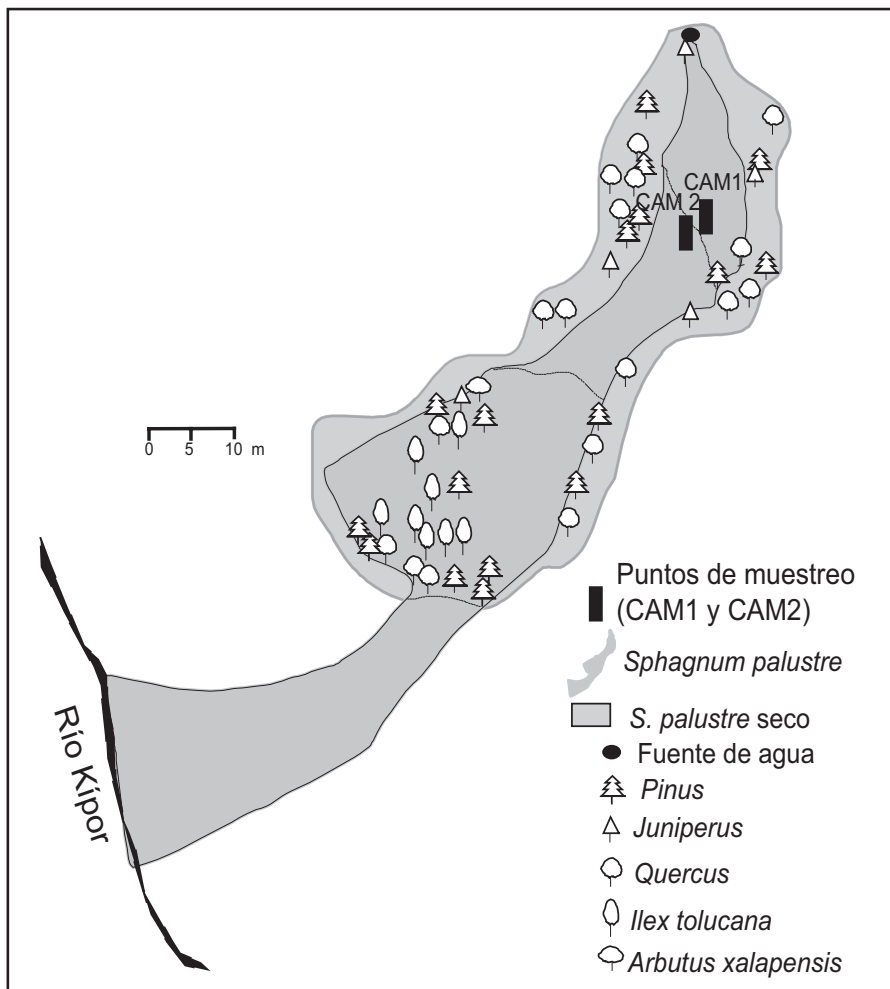

Fig. 2. Plano de la Ciénega de Camilo con indicación de la vegetación arbórea que crece en su interior y de la localización de los puntos de muestreo.

do del cañón aguas abajo, se desarrollan árboles riparios como Alnus oblongifolia, Cupressus arizonica, llex tolucana, Prunus gentryi y Juniperus mucronata. En la ciénega también crecen algunas especies de Poaceae (Agrostis scabra, Andropogon glomeratus, Panicum acuminatum) y Cyperaceae (Carex turbinata, Cyperus spp., Eleocharis spp., Juncus spp. y Rhynchospora sp.). Las pteridofitas incluyen a Polypodium polypodioides, Selaginella rupicola, S. novoleonensis, y ocasionalmente Athyrium filix-femina y Plagiogyria pectinata.

\section{MÉTODOS}

Se obtuvieron dos núcleos de sedimento, CAM1 y CAM2 (Fig. 2), con un nucleador de tipo Livingstone (Wright, 1991) en uno de los "escalones" de la turbera, a 


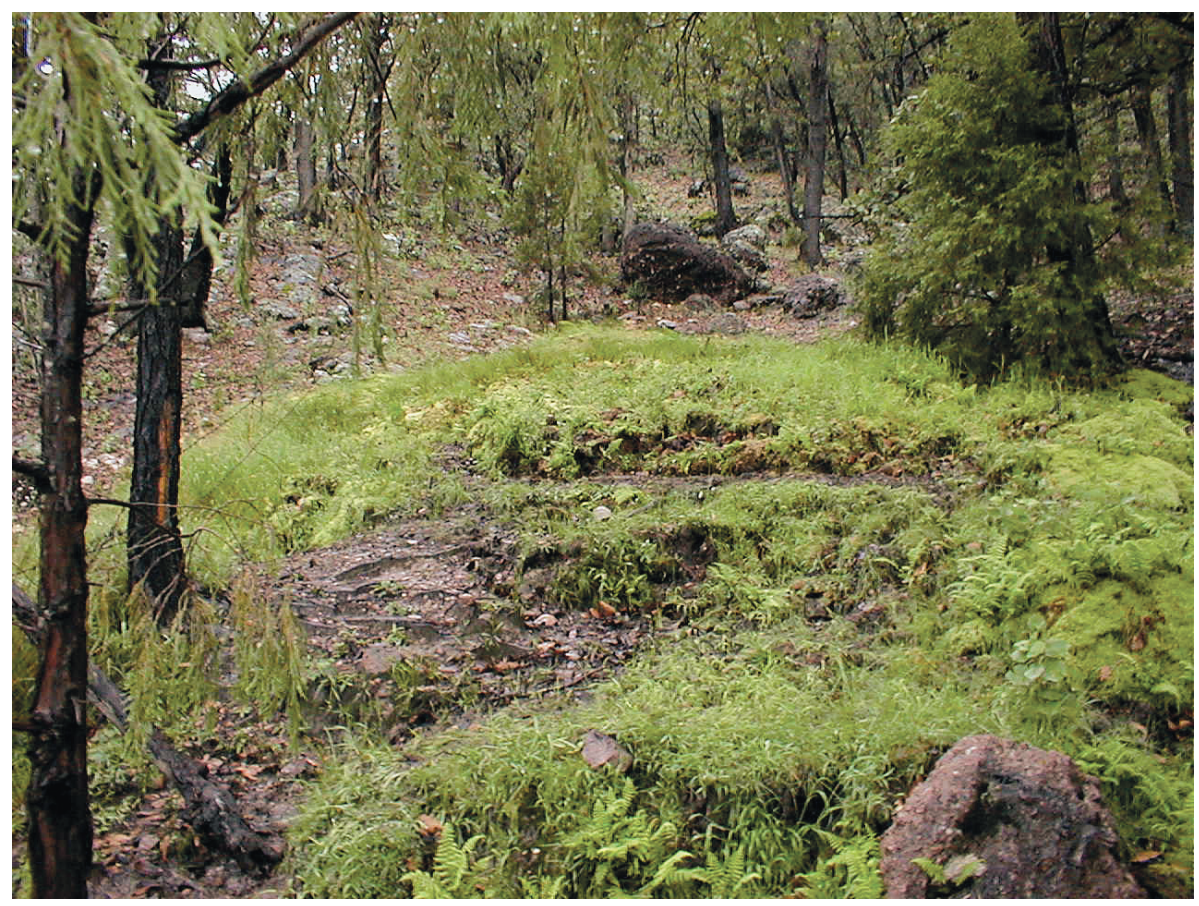

Fig. 3. Ciénega de Camilo, municipio de Yécora, Sonora. Fotografía de Carmen I. OrtegaRosas.

$25 \mathrm{~m}$ de distancia por debajo de la fuente de agua, distanciados $2 \mathrm{~m}$ uno de otro. Se tomaron muestras de $1 \mathrm{~cm}^{3}$ de sedimento y se trataron en laboratorio siguiendo métodos clásicos (Faegri et al., 1989): filtrado con tamiz de $500 \mu, \mathrm{HCl}, \mathrm{NaOH}, \mathrm{HF}$ en caliente, filtrado con malla de $7 \mu$ y acetólisis. Se añadieron pastillas de Lycopodium clavatum (batch 124961) para calcular la concentración polínica. Los porcentajes de polen y palinomorfos no polínicos (algas, hongos, bacterias y zooplancton) se refieren a la suma polínica total de cada nivel, y los porcentajes de esporas de helechos, a la suma total de polen y esporas. Se definieron tipos polínicos de Pinus según el tamaño del grano. Los palinomorfos no polínicos se indican con los nombres científicos correspondientes cuando se identifican como tales, o bien se presentan como tipos que en ocasiones pueden relacionarse con estructuras identificadas, o bien son incertae sedis. Los diagramas se graficaron con el programa Gpalwin (Goeury, 1997), el análisis de componentes principales de los datos polínicos se obtuvo con el programa PC-ORD 3.15; excluyéndose en este análisis los de palinomorfos no polínicos. La zonación de los diagramas de estos últimos se basa en las variaciones 
cualitativas de los taxones con mayores frecuencias y de los más representativos desde el punto de vista ecológico. Las dos dataciones radiométricas se obtuvieron en el laboratorio Beta Analytic Inc., en Florida, Estados Unidos, por el método radiométrico convencional y fueron calibradas con el programa CALIB 5.0.1 (Stuiver y Reimer, 1993); se presentan como edades calibradas antes del presente (cal. BP).

\section{RESULTADOS}

Estratigrafía y cronología

Los dos núcleos (CAM1 y CAM2) muestran arcillas orgánicas de color gris oscuro, fragmentos de Sphagnum palustre en descomposición en los niveles superiores (Figs. 4 y 5), y plantas vivas de Sphagnum palustre en la superficie del sitio. Adicionalmente, el núcleo CAM1 presenta en la base arcillas orgánicas de color gris claro que no se observan en CAM2. El Cuadro 1 indica las edades de radiocarbono obtenidas, con sus intervalos de edad y las fechas calibradas equivalentes antes del presente y después de Cristo. La base del núcleo CAM1 (67 a $54 \mathrm{~cm}$ de profundidad) fue fechada en $870 \pm 80 \mathrm{cal}$. BP. Sedimentos suprayacentes del mismo núcleo (43 a $30 \mathrm{~cm}$ de profundidad) dieron una edad de $1058 \pm 60 \mathrm{cal}$. BP. Las dos fechas son muy próximas teniendo en cuenta sus márgenes de error, pero se observa una ligera inversión que sugiere la existencia de una perturbación en la sedimentación, probablemente ligada a un deslizamiento de sedimentos. Por esta razón no se considera que el registro cubre los últimos mil años (hecho que se podría definir con un estudio muy minucioso de radiocarbono de los $30 \mathrm{~cm}$ superiores, lo que está fuera del objetivo de nuestro trabajo). Sin embargo, el intervalo entre 67 y $30 \mathrm{~cm}$ sí es representativo del período de alrededor de hace 1000 años que indican las dos dataciones, de la misma manera que el intervalo superior indica condiciones estratigráficas diferentes tanto en el tipo de sedimento como en el contenido de microfósiles.

\section{Análisis polínico}

Las figuras 4 y 5 ilustran los resultados de los análisis polínicos de los dos núcleos. Puede observarse la disminución de las frecuencias polínicas de Pinus y de Pteridophyta (estas últimas más variables) hacia la parte superior de las secuencias, y un ligero incremento de las de Quercus y Cupressaceae. La Fig. 6 muestra la definición y correlación de zonas polínicas por el análisis de componentes principales 


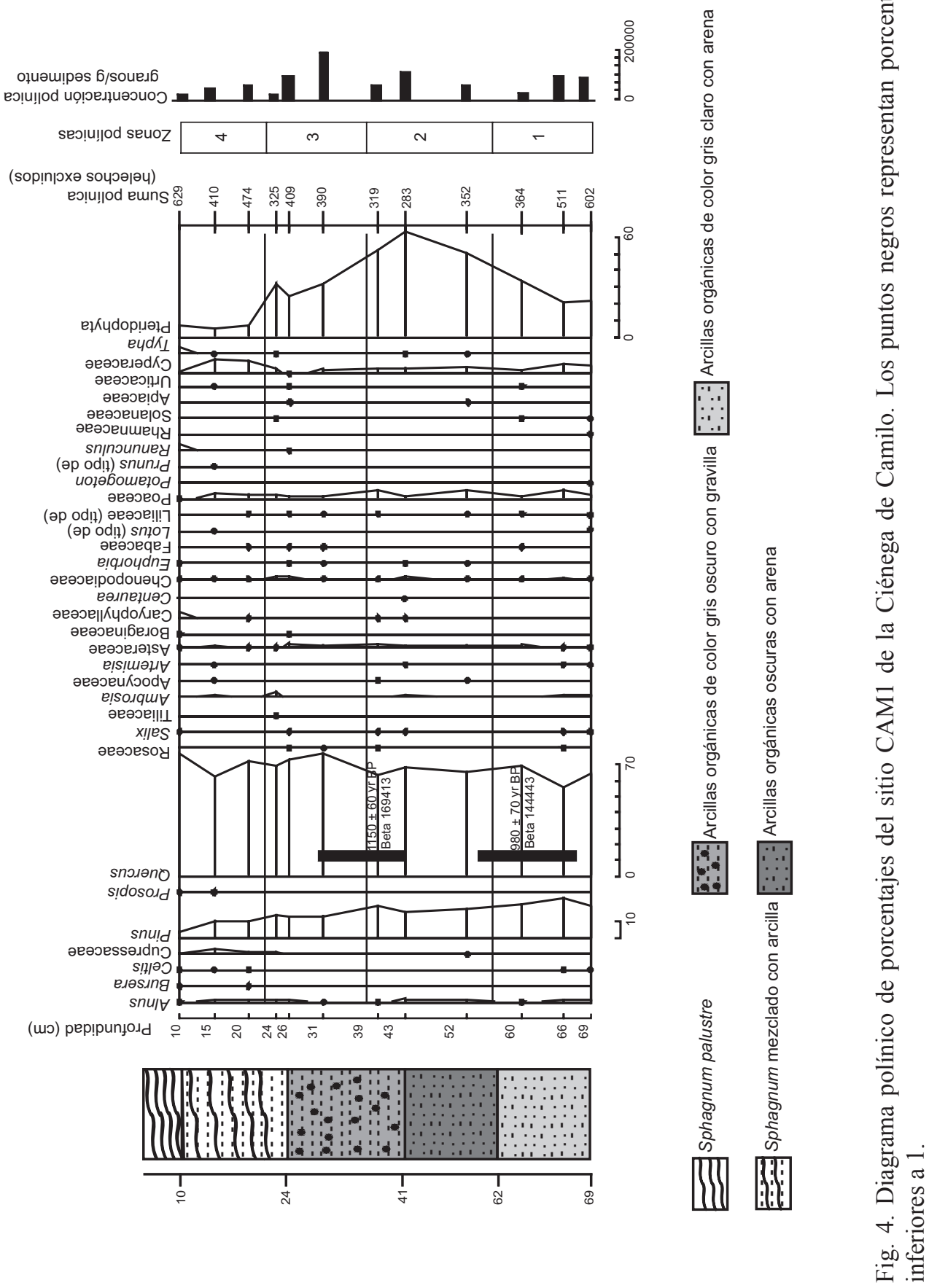


8
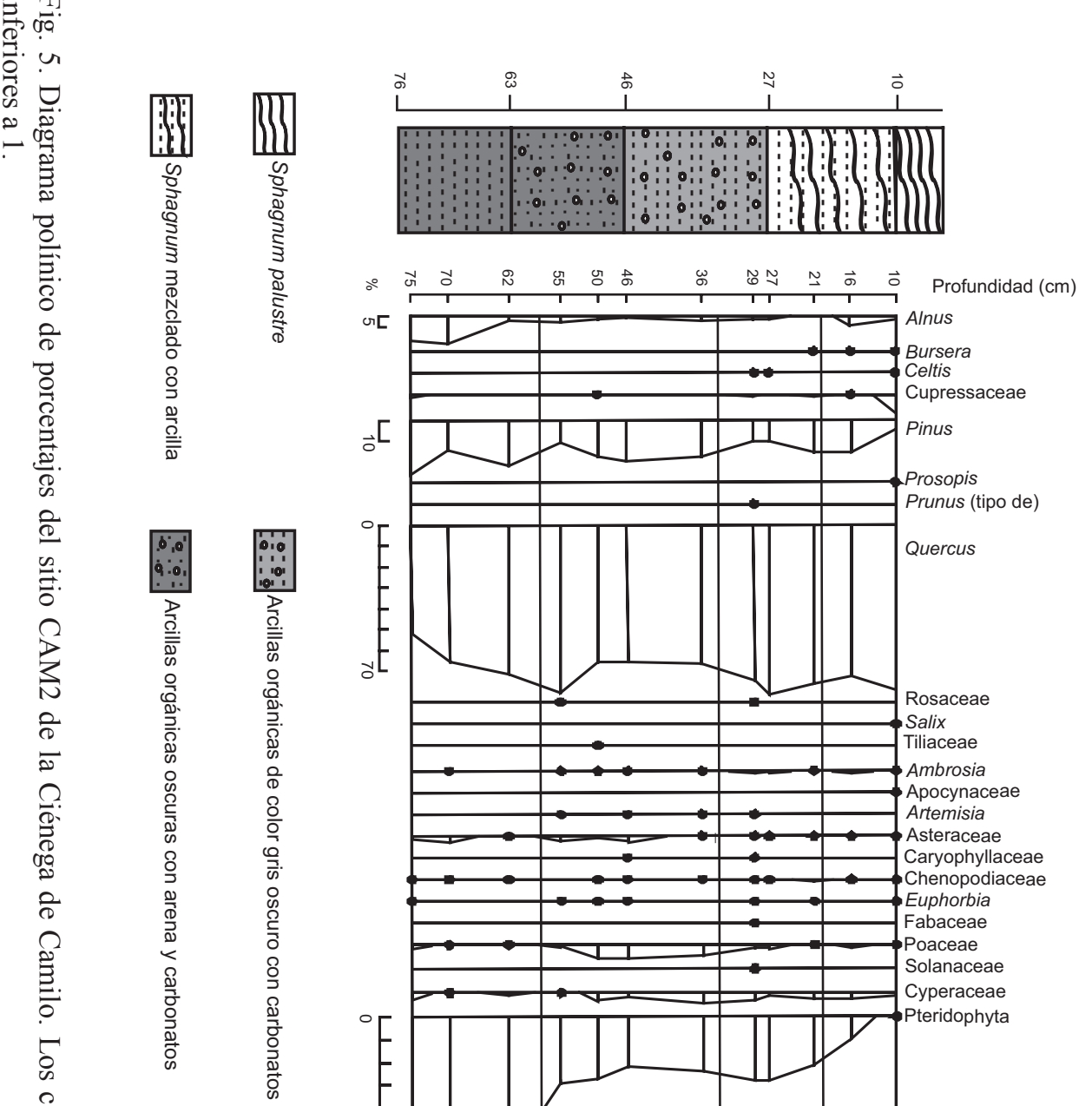

${ }_{0}[$
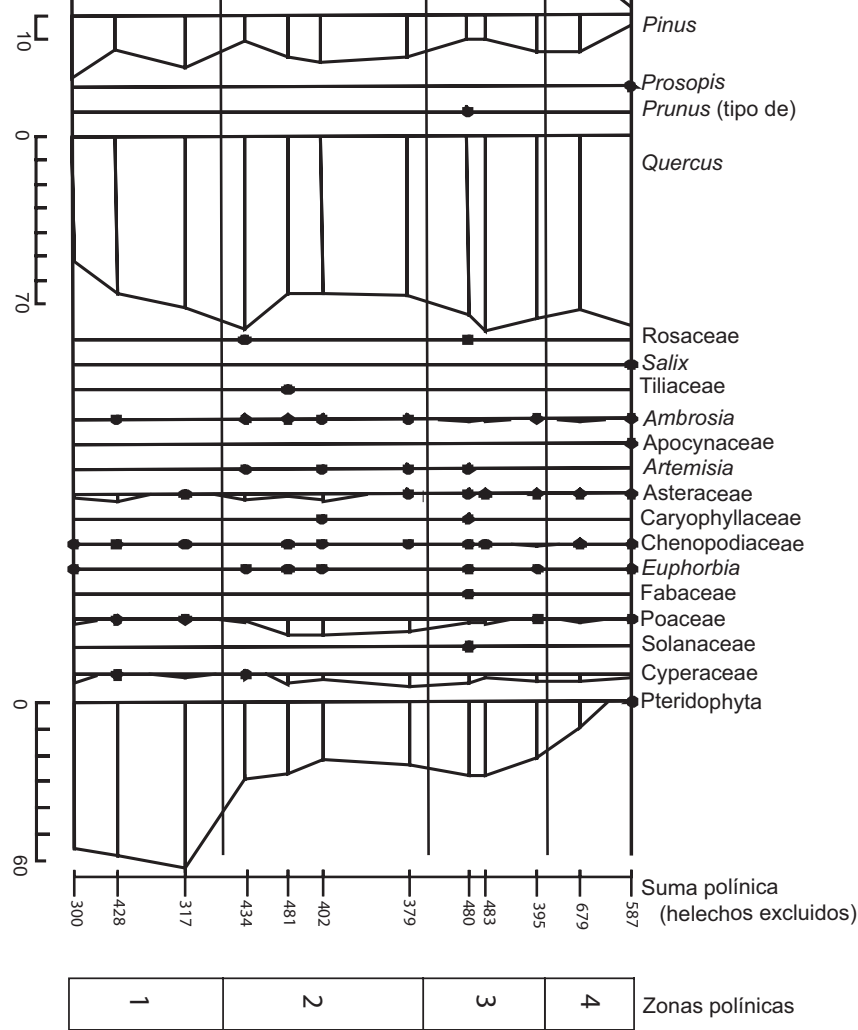
Cuadro 1. Fechas de radiocarbono, intervalos de edad y fechas calibradas equivalentes antes del presente (BP) y después de Cristo (AD). La calibración fue hecha con el programa CALIB 5.0.1 (Stuiver y Reimer, 1993).

\begin{tabular}{ccccccc}
\hline $\begin{array}{c}\text { CAM1 } \\
\text { (profundidad } \\
\mathrm{cm})\end{array}$ & $\begin{array}{c}\text { Número de } \\
\text { laboratorio }\end{array}$ & $\begin{array}{c}\text { Fecha } \\
\left(\text { años }{ }^{14} \mathrm{C}\right. \\
\mathrm{BP})\end{array}$ & $\begin{array}{c}\text { Intervalo } \\
\text { con dos } \\
\text { sigmas } \\
\text { (años cal } \\
\text { AD) }\end{array}$ & Probabilidad & $\begin{array}{c}\text { Fecha } \\
\text { calibrada } \\
\text { (años AD) }\end{array}$ & $\begin{array}{c}\text { Fecha } \\
\text { calibrada } \\
\text { (años BP) }\end{array}$ \\
\hline $30-43 \mathrm{~cm}$ & Beta 169413 & $1150 \pm 60$ & $768-1015$ & 0.96 & 891.5 & 1058.5 \\
$54-67 \mathrm{~cm}$ & Beta 144443 & $980 \pm 70$ & $946-1214$ & 0.97 & 1080 & 870 \\
\hline
\end{tabular}

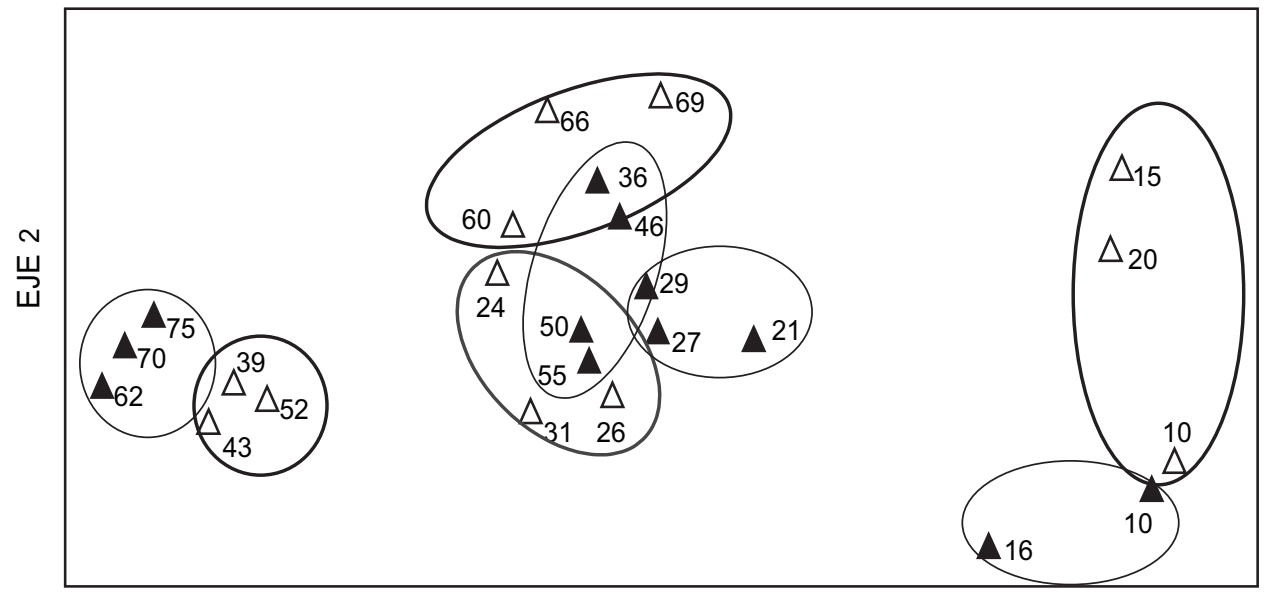

EJE 1

Fig. 6. Diagrama del análisis de componentes principales (ACP) de las muestras polínicas de $\mathrm{CAM} 1$ y CAM2. CAM1 $=\Delta$ y CAM2 $=\boldsymbol{\Delta}$. Los números indican la profundidad en $\mathrm{cm} . \mathrm{El}$ eje 1 explica $82 \%$ de la varianza total y el eje $2,14 \%$.

(ACP, únicamente basado en los granos de polen). El primer componente está correlacionado positivamente con Pteridophyta y Pinus, y negativamente con Quercus y Cupressaceae. El segundo está dominado por valores fuertemente positivos de Poaceae, Cyperaceae, Pinus y Chenopodiaceae, así como valores intensamente negativos para Quercus, Pteridophyta, Alnus y Cupressaceae. Los dos primeros componentes explican $96.5 \%$ de la varianza total. 
Se definieron y correlacionaron mediante el ACP cuatro zonas polínicas para CAM1 y cuatro para CAM2 (Fig. 6, Cuadro 2). En la primera (CAM1-1), Quercus (70\% de la suma polínica) es el taxon dominante. El polen de Pinus alcanza las mayores frecuencias de la secuencia (25\%), lo que sugiere la mayor abundancia (y/o mayor producción polínica) de árboles de pino dentro del bosque de pino y encino. Las zonas CAM1-2 y CAM2-1 se caracterizan por los altos porcentajes de polen de Quercus (70\%), un ligero descenso de los de Pinus (ca. 20\%), aumento de frecuencias de esporas de Pteridophyta (hasta 64\%), y $\geq 10 \%$ Alnus (probablemente A. oblongifolia). Los Pteridophyta corresponden mayormente a esporas monoletes desprovistas de perisporio, que probablemente incluyen las familias Aspleniaceae, Dryopteridaceae y Polypodiaceae. Asplenium palmeri, Athyrium filix-femina y Polypodium polypodioides están presentes en el sitio en la actualidad (Van Devender et al., 2003). Las altas frecuencias polínicas de las esporas son indicativas de poblaciones mayores que las actuales.

Las zonas CAM1-3, CAM2-2 y CAM2-3 muestran un descenso de las frecuencias de Pteridophyta a $30 \%$ y de Pinus a $12-16 \%$. Quercus alcanza un máximo de $77 \%$ y permanece dominante. Son notables Poaceae y Cyperaceae $(\leq 5 \%)$, particularmente en CAM2, donde probablemente sus altos porcentajes están ligados a la disminución de los valores de Quercus. Las zonas superiores CAM1-4 y CAM2-4 muestran valores altos de Quercus (80\%), de nuevo una disminución de Pinus (5\%) y Pteridophyta (hasta casi desaparecer), así como un aumento del polen de Cupressaceae $(\leq 10 \%)$, Cyperaceae $(\leq 10 \%)$ y Typha. La presencia de poblaciones incipientes de Typha se confirma por las ascosporas del tipo 206 (ver palinomorfos no polínicos, Figs. 7 y 8$)$.

Análisis de palinomorfos no polínicos (PNP)

Los palinomorfos no polínicos (Figs. 7 y 8, Fig. 9) son raros en la base de las secuencias (zonas de PNP 1 y 2), probablemente debido a la naturaleza arcillosa del sedimento que puede haber limitado su conservación. Sin embargo, la observación de espermatóforos de copépodos (tipo 28; 2\%) indica la presencia, aun cuando temporal, de aguas abiertas (Van Geel, 1978) durante la zona 1, lo que sugiere que la humedad edáfica fue mayor hace alrededor de $1000 \mathrm{BP}$ en el inicio de la sedimentación, que en los demás períodos. Entre otros PNP se incluyen amerosporas indiferenciadas de hongos, Spirogyra (tipo 130), esporas de musgos, el tipo 55A (esporas de Sordariaceae), y ocasionalmente Zygnema (tipo 314). Este conjunto indica un período relativamente seco y aguas estancadas eu- a mesotróficas. 
Cuadro 2. Correspondencia entre las zonas polínicas de los núcleos CAM 1 y CAM 2 de la Ciénega de Camilo.

\begin{tabular}{|c|c|}
\hline Zonas polínicas CAM1 & Zonas polínicas CAM2 \\
\hline 4 & 4 \\
\hline 3 & 3 \\
\hline 2 & 2 \\
\hline 1 & 1 \\
\hline
\end{tabular}

Los PNP son abundantes y más diversos en los niveles superiores con Sphagnum (zonas 3 y 4). En la 3, las amerosporas indiferenciadas, Pediastrum (tipo 900) y las cianobacterias Anabaena y Aphanizomenon alcanzan sus valores máximos que indican una intensificación del período árido, una máxima concentración de nutrientes en el medio ambiente acuático mesotrófico, y posiblemente la presencia de ganado o agricultura. Pleospora (tipo 3B) y el tipo 82E reflejan un clima árido y condiciones ombrotróficas en el sitio (sedimento depositado únicamente por el efecto del agua de lluvia).

La zona 4 de PNP se caracteriza por los mismos tipos dominantes de la zona 3, pero con menores frecuencias, y porque el tipo 55A y Anabaena desaparecen del registro. Sin embargo, se observa un incremento en las frecuencias de los tipos 14C, $22 \mathrm{~A}, 126$, y se registran las primeras apariciones de los tipos 72D, 88A, 93, 332E, 425, el tipo 36 (Acari, Oribatei) y Cosmarium cf. controversum. El registro de ácaros es indicador de cambios florísticos y podría relacionarse en el polen con el aumento de Cupressaceae y Alnus, así como la disminución de Pinus y Pteridophyta. También están presentes las amerosporas indiferenciadas de hongos, Anabaena, Pediastrum, Spirogyra, Aphanizomenon gracile, y conidios de Sporidesmium sp. Estos conjuntos sugieren de nuevo un ambiente mesotrófico ombrotrófico, probablemente algo más húmedo que el de los niveles subyacentes. Lo anterior concuerda con el aumento de esporas del tipo 3B (Pleospora) y de Sphagnum, un género de musgos típico de humedales ombrogénicos.

Para CAM2, las zonas polínicas y de PNP coinciden, pero no es así para CAM1, posiblemente porque los cambios en el contenido polínico del sedimento están más relacionados con la vegetación regional, mientras que los cambios en PNP reflejan condiciones locales en el sitio. 


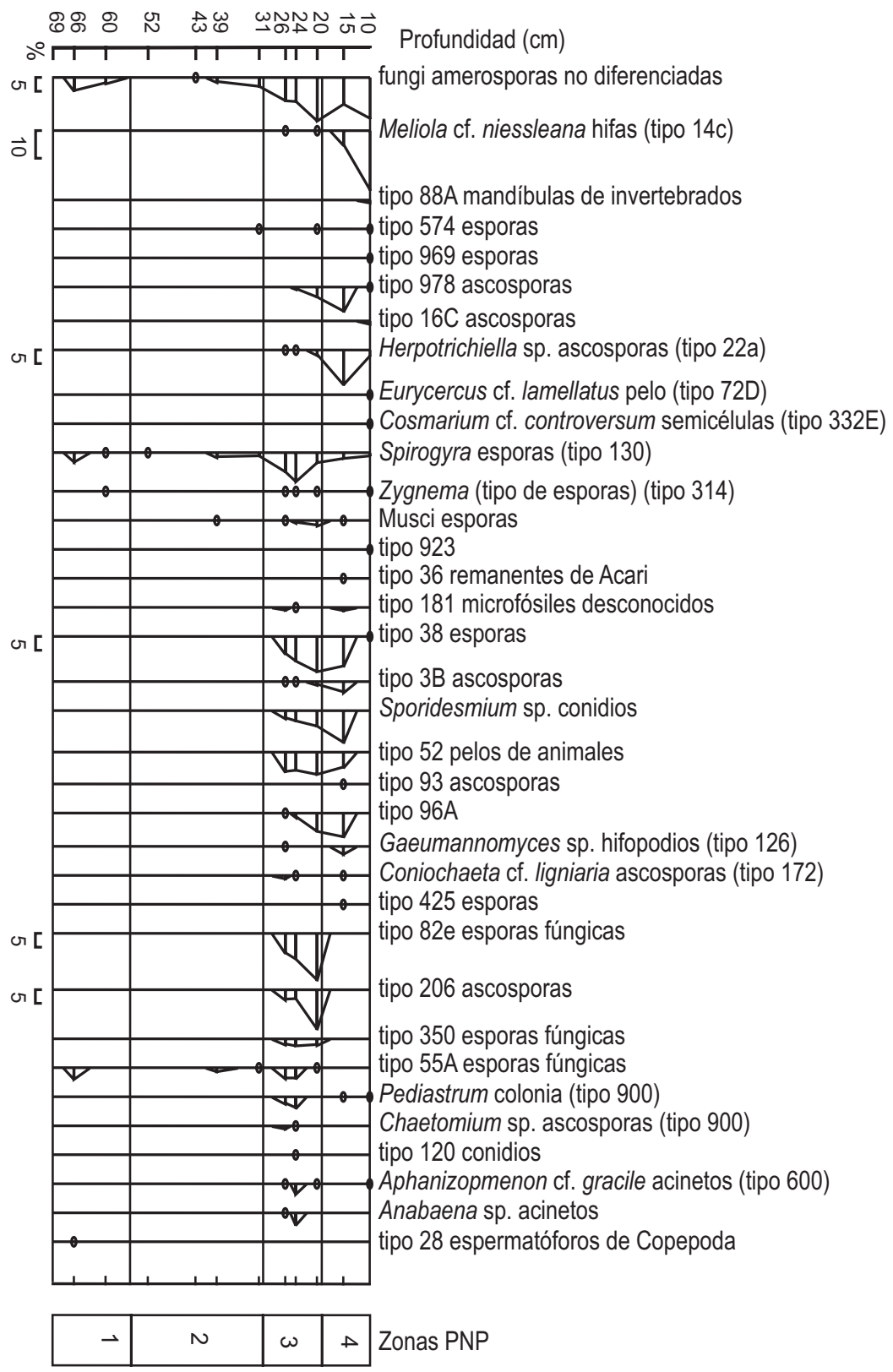

Fig. 7. Diagrama de porcentajes de palinomorfos no polínicos (PNP) del sitio CAM1 de la Ciénega de Camilo. Los óvalos representan porcentajes inferiores a 1. 
Ortega-Rosas et al.: Retrospectiva del bosque de pino y encino de la Sierra Madre Occidental

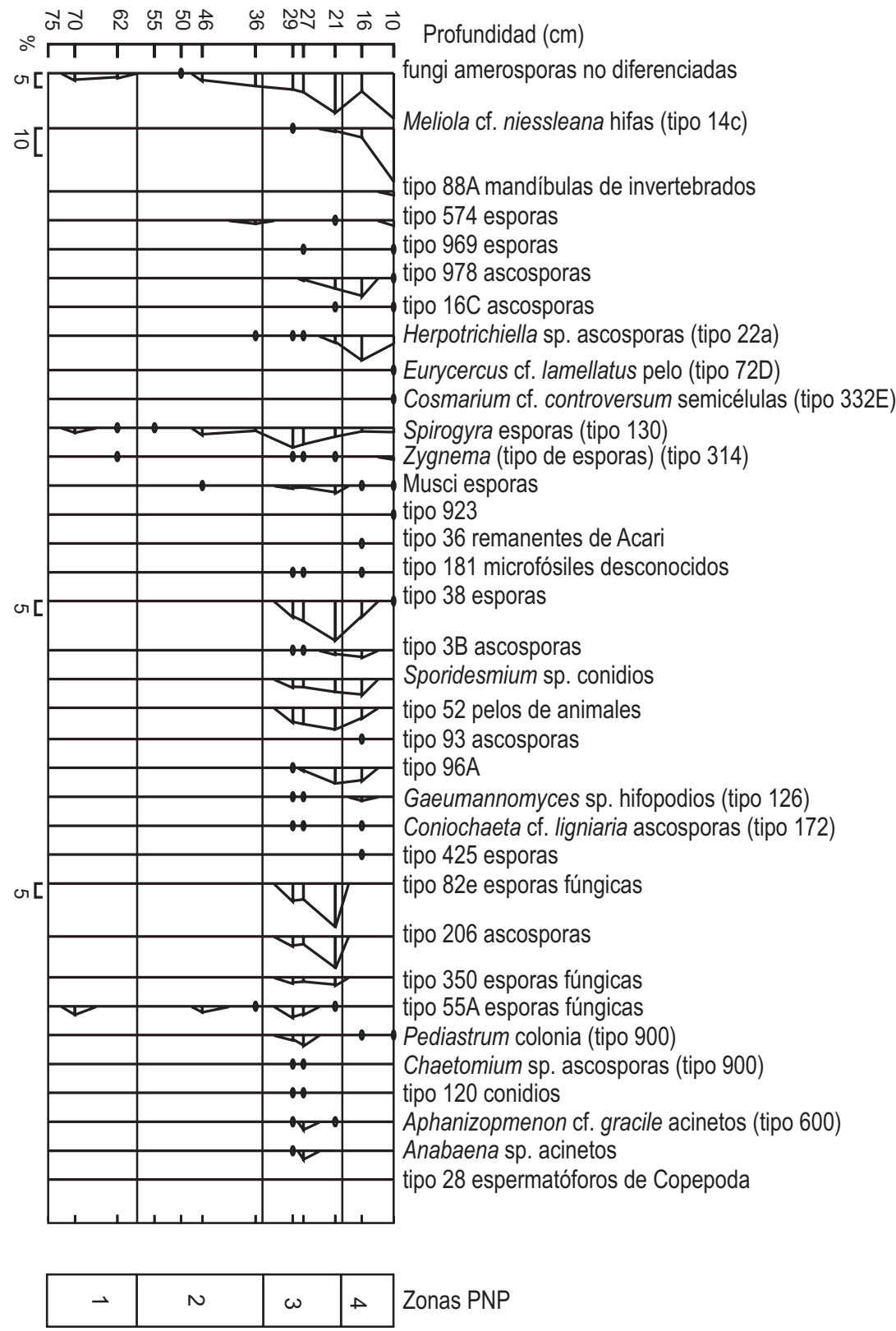

Fig. 8. Diagrama de porcentajes de palinomorfos no polínicos (PNP) del sitio CAM2 de la Ciénega de Camilo. Los óvalos negros representan porcentajes inferiores a 1. 

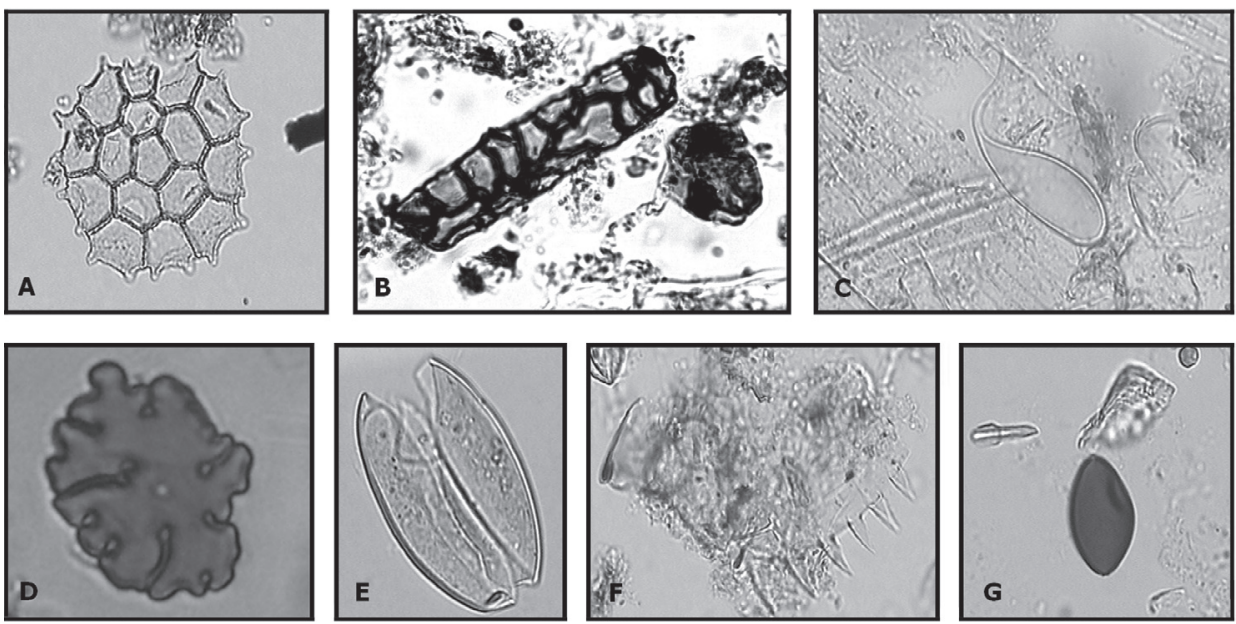

Fig. 9. Ejemplos de algunos palinomorfos no polínicos. A: Pediastrum, B: Pleospora, C: tipo 28, D: tipo 126, E: Spirogyra, F: tipo 72D, G: tipo 55A. Fotografías de J. Antonio LópezSáez.

\section{DISCUSIÓN}

Estratigrafía y cronología

La ligera inversión de las fechas obtenidas en el núcleo CAM1 (870 \pm 80 cal. BP y $1058 \pm 60$ cal. BP, Fig. 3) puede interpretarse de dos maneras que apuntan hacia una misma causa. En primer lugar, teniendo en cuenta los márgenes de error de las dos fechas, la diferencia entre las edades es de 48 años, cantidad estadísticamente poco significativa. Puede entenderse que todo el sedimento comprendido entre la base y el nivel de $30 \mathrm{~cm}$ fue depositado alrededor de hace 1000 años, indicando una tasa de sedimentación relativamente elevada si la comparamos con la profundidad total de la secuencia y su edad. Sólo la existencia de un clima húmedo permitiría tal sedimentación.

La segunda interpretación considera que existe una inversión real, y que los niveles comprendidos entre 43 y $30 \mathrm{~cm}(1058 \pm 60$ cal. BP) son ligeramente más antiguos que la base, originalmente depositados en un lugar superior de la ciénega, más próximo a la fuente de agua, y que sufrieron un deslizamiento y se redepositaron sobre los niveles de CAM1 fechados en $870 \pm 80$ cal. BP. Este deslizamiento también sólo pudo darse bajo la existencia de un clima húmedo. 
La estratigrafía polínica descrita más arriba no detecta cambios importantes atribuibles a esta última causa; sin embargo, en el análisis microscópico se observó una mayor degradación de los granos de polen en los niveles comprendidos entre 43 y $30 \mathrm{~cm}$, calculándose de 35 a 38\% de microsporas deterioradas de Quercus (taxon dominante en toda la secuencia) frente a 8 a $20 \%$ observados en el resto de la columna. Por otra parte, en estos niveles se aprecia la presencia de gravilla, que sugiere la influencia de una erosión. La degradación del polen y la presencia de gravilla son argumentos a favor de la hipótesis de un deslizamiento.

Los niveles entre los $30 \mathrm{~cm}$ de profundidad y la superficie son litológicamente diferentes, dominando en ellos el esfagno (acumulación de restos de Sphagnum). Representan una época más reciente y denotan, a través de los PNP, condiciones de mayor aridez en el período posterior a $1000 \mathrm{BP}$.

Cambios ambientales bióticos

Las frecuencias polínicas de Pinus y Quercus en CAM1 y CAM2 se compararon con las de muestras de lluvia polínica actual de la región (Ortiz-Acosta, 2003; Ortega-Rosas, 2003). En el bosque de pino y encino los valores porcentuales de polen de Quercus son elevados y los de Pinus pueden alcanzar $30 \%$ en lugares en que la densidad de pinos es mayor, pero son inferiores, como en nuestro caso, cuando los pinos son menos abundantes, pues así como los pinos son buenos productores polínicos, también los encinos lo son. Factores como la orientación sur de una ladera son a menudo determinantes de la baja proporción de polen de pinos. En consecuencia, la comparación sugiere que hace 1000 años el sitio estuvo cubierto por una vegetación de bosque de pino y encino. Sin embargo, el polen y los PNP registraron cambios significativos en su composición. Éstos se reflejaron en una disminución progresiva de la relación Pinus/Quercus y una cada vez menor abundancia y diversidad de esporas de helechos hacia los niveles más recientes (Figs. 4 y 5). Dado que las frecuencias polínicas de Pinus, así como la precipitación, aumentan con la altitud en la región (Ortiz-Acosta, 2003; Ortega-Rosas, 2003), ello podría reflejar ambientes más húmedos y/o más fríos hace aproximadamente 1000 años.

El análisis morfológico de los granos de polen de Pinus muestra que hubo un cambio en las especies de pinos que poblaron la región. En la vegetación actual, Pinus chihuahuana, P. engelmannii, $P$. herrerae y $P$. yecorensis crecen en los alrededores del sitio. Hace aproximadamente 1000 años BP, la(s) especie(s) dominante era otra, cuyos granos de polen son de tamaño mayor (Fig. 10). Es posible que dicho árbol dominante corresponda a Pinus strobiformis, de microspora más grande y que 
actualmente crece a mayor altitud y en ambientes más húmedos y más fríos en la Mesa del Campanero, al oeste de Yécora. En cambio, la reducción de porcentajes de esporas de helechos y el aumento de los de polen de Cyperaceae y Poaceae observados en el sitio CAM2, zona 2, ocasionaron una disminución relativa en la contribución polínica de Quercus. También se registra un ligero aumento de amerosporas, por lo que el conjunto de estos cambios puede indicar un clima más árido.

El bosque y la ciénega experimentaron recientemente un cambio que viene dado por la disminución y desaparición final de los pinos con granos de polen grandes que ocurre al final de la zona 3 (CAM1-26 cm, Fig. 10) y por cambios en los PNP que se vuelven más abundantes y diversos de ahí en adelante (cambio de la zona 2 a la zona 3 de PNP, Figs. 7 y 8). Tanto amerosporas como Zygnemataceae, el tipo 55A y Pediastrum sugieren una creciente aridez local. Es más, Pleospora y el tipo 82e indican un medio ombrotrófico (Van Geel, 1978) que precede al establecimiento de Sphagnum (Figs. 4 y 5). La aparición de este último se debe probablemente a una dispersión y germinación exitosa de sus esporas en un sustrato favorable de un nuevo lugar de la ciénega. Sabemos, por el estudio de otro núcleo de sedimento de la misma ciénega, que Sphagnum está presente en la ciénega desde hace al menos 6000 BP (Ortega-Rosas, 2003).

Los PNP sugieren que en todo el período cubierto por el registro existieron la fuente de agua y el curso de agua permanente, aunque variaron tanto la superficie del espejo de agua como el área de ciénega. Hace 1000 años, el área de la terraza de la ciénega donde se localizaron los dos sitios cenagosos de sondeo que hoy son emergentes, estuvo cubierta de agua.

Desde un punto de vista trófico, los PNP sugieren que los sitios CAM1 y CAM2 eran eu- a mesotróficos en las fases más antiguas, con aguas abiertas y estancadas, y mesotróficos a ombrotróficos en las fases más recientes (zonas 3 y 4). De este modo, la ciénega siguió un proceso de desarrollo similar al de las turberas de latitudes más elevadas (Mitsch y Gosselink, 2000), incluyendo un progresivo aislamiento hidrológico del flujo de agua subterránea, siendo inicialmente minerotrófica-mesotrófica, alimentada por el agua del subsuelo y la escorrentía superficial, y finalmente en algunos lugares ombrotrófica, alimentada por la precipitación.

Cambios ambientales abióticos

La sedimentación inició alrededor de 1000 cal. BP en el sitio de sondeo CAM1, lo que implica que en ese momento sucedió un cambio climático favorable al desarrollo y acumulación de arcillas basales que dieron lugar posteriormente al establecimiento de la ciénega. 


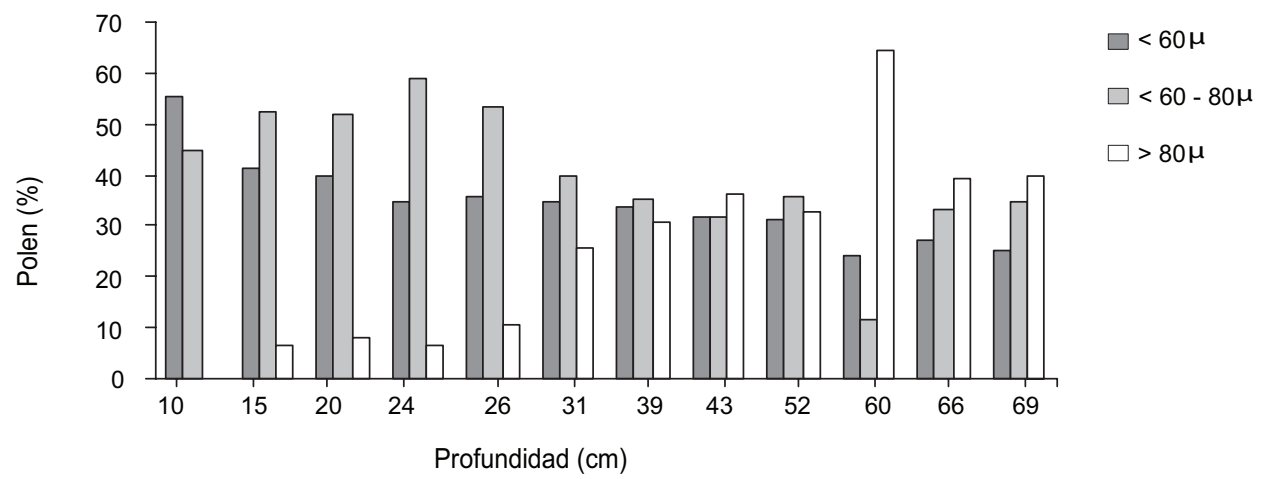

Fig. 10. Tamaño de los granos de polen de Pinus en CAM1. Los porcentajes están referidos al total de polen de Pinus.

En lugares cálidos, el desarrollo de ciénegas depende de la persistencia de un ambiente húmedo durante todo el año (Mitsch y Gosselink, 2000). Actualmente, la falta del agua es un factor limitante en abril y mayo por la escasa precipitación; en verano, los intensos aguaceros monzónicos pueden compensar la evapotranspiración moderada del bosque. Hace 1000 años, las lluvias de primavera, y probablemente también las de verano, debieron ser mayores que las de las épocas anteriores, lo que permitió que prevaleciera una humedad más continua a lo largo de todo el año, que a su vez favoreció el inicio del desarrollo de la sedimentación en esta zona de la turbera. El clima relativamente húmedo persistió un tiempo, ya que la ciénega se amplió, iniciándose la sedimentación en el sitio CAM2. Las zonas polínicas CAM1-2 y CAM2-1 reflejan aún la existencia de un ambiente húmedo (Pinus, Pteridophyta), pero los PNP indican que se trata de un período relativamente seco, ya que se encuentran amerosporas sin identificar (López-Sáez et al., 2002), es decir, el clima fue probablemente algo menos húmedo que en la zona CAM1-1.

\section{Antropización}

No se han encontrado evidencias directas de disturbio humano, como pudiera ser el polen del maíz. La familia Cupressaceae se ha relacionado en algunos casos con la sucesión secundaria y la acción antrópica (Rzedowski, 1981, p. 311; Rousset y Lepart, 1999), y los valores altos de Cupressaceae de los niveles más recientes (zona polínica 4) podrían corresponder a la disminución de la producción polínica de pino, en el sentido de que un clareo del bosque de pino y encino puede haber estado seguido de la expansión de Juniperus deppeana. Pero el polen de Cupressaceae 
también podría corresponder al de otras dos especies que crecen en el sitio actualmente: Juniperus mucronata y Cupressus arizonica, ambos árboles riparios, cuyo incremento cabría relacionar más con un cambio climático a mayor humedad más que un cambio de carácter antrópico.

En los niveles más recientes de la secuencia de PNP, las cianobacterias Aphanizomenon cf. gracile y Anabaena que están relacionadas con altos niveles de nitrógeno asociados a presencia de ganado o agricultura (Van Geel et al., 1994, 1996), así como el tipo 55A (Sordariaceae), constituyen indicadores antrópicos. Tanto los indios Pimas como posiblemente otros grupos indígenas han habitado la región por cientos o miles de años, y aunque su impacto en la vegetación fue inicialmente débil, seguramente llegó a ser más fuerte después del establecimiento de la misión jesuita cercana de Maycoba, en el siglo XVII (Beaumont Pfeifer y Duarte Rodríguez, 2001). Más recientemente, la acción humana ha tenido un impacto mucho mayor en los bosques de pino y encino del área de Yécora-Maycoba. Desde 1963, los pinos han sido talados para obtener madera (Ortiz-Garay, 1995), y la extracción de encinos comenzó en 1997, el mismo año en que se muestreó el núcleo de sedimento de la ciénega de Camilo; estos últimos cambios por tanto no se registraron en nuestros sedimentos.

\section{CONCLUSIONES}

El inicio de la sedimentación en CAM1 data de alrededor de 1000 cal. BP. Un cambio a condiciones climáticas más húmedas en ese momento fue necesario para dar inicio a la acumulación de material orgánico en ese punto de la ciénega. Es posible que las temperaturas fueran también inferiores, disminuyendo las pérdidas de agua por evaporación. La comparación con el clima actual de la región sugiere que las primaveras y posiblemente también los veranos debieron haber sido más húmedos. Lo anterior concuerda con la apreciación de Van Devender (1987) sobre un intervalo húmedo en el Holoceno final, alrededor de 980 y 990 BP con considerable lluvia en ambas estaciones, la fría y la cálida.

Como resultado de ello, los pinos fueron más abundantes que en la actualidad en el bosque de pino y encino que rodeaba la Ciénega de Camilo. Estaban presentes cerca del sitio otras especies, que posiblemente incluyeron a Pinus strobiformis, que actualmente crece a mayor elevación, en hábitats más húmedos. El sotobosque herbáceo y la vegetación de la ciénega incluían principalmente a Poaceae, Cyperaceae y Pteridophyta, como hoy; este último grupo, que hoy día es ocasional aunque 
integrado por seis familias (Van Devender et al., 2003), fue más abundante en el pasado. El lapso húmedo alrededor de $1000 \mathrm{BP}$ corresponde al período arqueológico de desarrollo de los asentamientos de las culturas de Sonora y Chihuahua. Por el contrario, es interesante observar que en el sureste del país esta época corresponde a la denominada "sequía maya" del final del periodo Clásico. En efecto, el antagonismo entre las tendencias climáticas del noroeste y sureste del país ha sido puesto en evidencia no sólo en el clima actual, sino también en estudios paleoclimáticos en los registros del final del Pleistoceno y del Holoceno (Metcalfe et al., 2000).

Los niveles suprayacentes a los fechados registran condiciones más áridas definidas por la disminución del polen de pino y cambios en los PNP (amerosporas, Zygnemataceae, tipo 55A y Pediastrum), que también muestran la evolución del sitio de condiciones eutróficas-mesotróficas a ombrotróficas (Pleospora, tipo 82E y Sphagnum). A pesar de estar localizada en el límite sur de la distribución de las turberas de Sphagnum, la Ciénega de Camilo parece haber seguido los mismos procesos de desarrollo que las caracterizan en latitudes de clima templado y boreales.

En el nivel más reciente, los palinomorfos no polínicos sugieren condiciones ligeramente más húmedas, aunque el polen -a excepción de la presencia de Typhano lo refleja particularmente. Quizá tal relativa humedad es la que ha favorecido la conservación de este hábitat de ciénega tan particular para la región.

\section{AGRADECIMIENTOS}

La Dirección de Intercambio Académico y la Coordinación de la Investigación Científica, ambas pertenecientes a la Universidad Nacional Autónoma de México, y la fundación "María Bueno" proveyeron de fondos y becas a los autores Carmen Isela Ortega-Rosas y José Antonio López-Sáez. El trabajo de campo fue financiado por el Instituto de Ecología de la Universidad Nacional Autónoma de México, al que pertenecía la autora María Cristina Peñalba. Alfonso Valiente revisó una versión previa del manuscrito. La extracción polínica se llevó a cabo en el laboratorio de química del Instituto de Geología, Universidad Nacional Autónoma de México, Estación Regional Noroeste. Diego Pérez supervisó el ACP. Un agradecimiento especial se debe a Luis Coyote, jefe de la comunidad Pima de El Kípor, Sonora, por su ayuda en el campo. Gracias también a Don Néstor Aguilar y su esposa Doña Rosalía por permitirnos el acceso a la ciénega. Lizeth Larios, Martín Ortiz, Francisco Paz y Ana Lilia Reina colaboraron en el trabajo de campo y laboratorio. 


\section{LITERATURA CITADA}

Álvarez-Palma, A. M. 1996. Sociedades Agrícolas. Historia General de Sonora. Período prehistórico y prehispánico. Tomo I. Gobierno del Estado de Sonora. Hermosillo, México. pp. 197-231.

Anderson, R. S. y D. S. Shafer. 1991. Holocene biogeography of spruce-fir forests in southeastern Arizona. Implications for the endangered Mt. Graham red squirrel. Madroño 38: 287-295.

Anderson, R. S. y T. R. Van Devender. 1995. Vegetation history and paleoclimates of the coastal lowlands of Sonora, Mexico. Pollen records from packrat middens. J. Arid Environ. 30: 295-306.

Beaumont Pfeifer, D. J. y B. A. Duarte Rodríguez. 2001. Los Pimas, Catálogo de piezas arqueológicas pertenecientes a la zona indígena pima. Gobierno del Estado de Sonora. Hermosillo, México. 44 pp.

Búrquez, A., A. Martínez-Yrízar y P. S. Martin. 1992. From the high Sierra Madre to the coast: changes in vegetation along highway 16 , Maycoba-Hermosillo. In: Clark, K. F., J. Roldán y R. H. Schmidt (eds.). Geology and resources in Northern Sierra Madre Occidental, Mexico. El Paso Geol. Soc. El Paso, Texas. pp. 239-252.

Cochemé, J. J. y A. Demant. 1991. Geology of the Yécora area, northern Sierra Madre Occidental, Mexico. Geological Society of America Special Paper 254: 81-94.

Davis, O. K., T. Minckley, T. Moutoux, T. Jull y B. Kalin. 2002. The transformation of Sonoran Desert wetlands following the historic decrease of burning. J. Arid Environ. 150(3): 393-412.

DiPeso, C. C. 1966. Archeology and ethnohistory of the Northern Sierra. In: Ekholm, G. F. y G. R. Willey (eds.). Handbook of Middle American Indians. Vol. 4. University of Texas Press. Austin, Texas. pp. 3-25.

Faegri, K., P. E. Kaland y K. Krzywinski. 1989. Textbook of pollen analysis. 4th ed. John Wiley \& Sons, Inc. Nueva York. 328 pp.

Goeury, C. 1997. GpalWin: gestion, traitement et représentation des données de la paléoécologie. XV Symposium, Association des Palynologues de Langue Française. Lyon, France. 31 pp.

López-Sáez, J. A., B. Van Geel y M. Martín-Sánchez. 2002. Aplicación de los microfósiles no polínicos en palinología arqueológica. In: Oliveira, J. V. (ed.). Contributos das ciências e das technologias para a arqueologia da Península Ibérica. Actas do $3^{\circ}$ Congresso de Arqueología Peninsular. Vol. IX. Vila-Real, Portugal, setembro de 1999, ADECAP, Porto, Portugal. pp. 11-20.

Lozano-García, S., B. Ortega-Guerrero y S. Sosa-Nájera. 2002. Mid- to Late-Wisconsin pollen record of San Felipe basin, Baja California. Quaternary Res. 58(1): 84-92.

Martin, P. S. 1963. The last 10,000 years. A fossil pollen record of the American Southwest. The University of Arizona Press. Tucson, Arizona. 87 pp.

Metcalfe, S. E., S. L. O'Hara, M. Caballero y S. J. Davies. 2000. Records of late PleistoceneHolocene climatic change in Mexico - A review. Quaternary Sci. Rev. 19: 699-721. 
Metcalfe, S. E., A. Say, S. Black, R. McCulloch y S. L. O’Hara. 2002. Wet conditions during the last glaciation in the Chihuahuan Desert, Alta Babícora basin, México. Quaternary Res. 57(1): 91-101.

Mitsch, W. J. y J. G. Gosselink. 2000. Wetlands. 3a.ed. John Wiley \& Sons, Inc. Nueva York. 920 pp.

Ortega-Guerrero, B., M. Caballero, S. Lozano-García y M. O. Villanueva. 1999. Paleoenvironmental record of the last 70,000 yr in San Felipe basin, Sonora Desert, Mexico. Geofísica Internacional 38(3): 153-163.

Ortega-Ramírez, J., A. Valiente-Banuet, J. Urrutia-Fucugauchi, C. A. Mortera-Gutiérrez y G. Alvarado-Valdez. 1998. Paleoclimatic changes during the Late Pleistocene Holocene in Laguna Babícora, near the Chihuahuan Desert, México. Can. J. Earth Sci. 35(10): 1168-1179.

Ortega-Rosas, C. I. 2000. Historia de la vegetación en la Ciénega de Camilo durante el Holoceno, municipio de Yécora, Sonora, Sierra Madre Occidental. Tesis de licenciatura, Centro de Estudios Superiores del Estado de Sonora. Hermosillo, Sonora. $106 \mathrm{pp}$.

Ortega-Rosas, C. I. 2003. Palinología de la Ciénega de Camilo: datos para la historia de la vegetación y el clima del Holoceno medio y superior en el NW de la Sierra Madre Occidental, Sonora, México. Tesis de maestría. Universidad Nacional Autónoma de México. México D.F. 109 pp.

Ortiz-Acosta, M. E. 2003. Données et méthodes pour l'histoire de la végétation et du climat du nord-ouest du Mexique. Thése. Université d'Aix-Marseille III. Marseille, France. 147 pp.

Ortiz-Garay, A. 1995. Pimas. Etnografía contemporánea de los pueblos indígenas de México, región noroeste. Instituto Nacional Indigenista. México, D.F. pp. 293-366.

Rhode, D. 2002. Early Holocene Juniper woodland and chaparral taxa in the central Baja California Peninsula, Mexico. Quaternary Res. 57(1): 102-108.

Roldán, J. y K. F. Clark. 1992. An overview of the geology and mineral deposits of the northern Sierra Madre Occidental and adjacent areas. In: Clark, K. F., J. Roldán y R. H. Schmidt (eds.). Geology and mineral resources of the northern Sierra Madre Occidental, Mexico. El Paso Geol. Soc. Field Cont. Guidebook 24. El Paso, Texas. pp. 39-65.

Rousset, O. y J. Lepart. 1999. Shrub facilitation of Quercus humilis regeneration in succession on calcareous grasslands. J. Veg. Sci. 10: 493-502.

Rzedowski, J. 1981. Vegetación de México. Limusa. México, D.F. 432 pp.

Sankey, J. T., T. R. Van Devender y W. H. Clark. 2001. Late Holocene plants, Cataviña, Baja California. The Southwest. Nat. 46(1): 1-7.

Sirkin, L., S. Pedrin, G. Padilla y E. Díaz. 1994. Holocene vegetation and climate of Baja California Sur, México. Rev. Mex. Cienc. Geol. 11(1): 79-86.

Stuiver, M. y P. J. Reimer 1993. Extended 14C data base and revised CALIB 3.0 14C age calibration program. Radiocarbon 35: 215-230.

Swanson, E. y D. Walk. 1988. Mid-Tertiary silic volcanism in Chihuahua, Mexico. In: Clark, K. F., P. Goodell y J. Hoffer (eds.). Stratigraphy, tectonics and resources of parts of the Sierra Madre Occidental Province, Mexico. El Paso Geol. Soc. El Paso, Texas. pp. 229-239. 
Van Devender, T. R. 1987. Holocene vegetation and climate in the Puerto Blanco mountains, Southwestern Arizona. Quaternary Res. 27(1): 51-72.

Van Devender, T. R. 1990a. Late Quaternary vegetation and climate of the Chihuahuan Desert, United States and Mexico. In: Betancourt, J. L., T. R. Van Devender y P. S. Martin (eds.). Packrat Middens. The last 40,000 years of biotic change. The University of Arizona Press. Tucson, Arizona. pp.105-133.

Van Devender, T. R. 1990b. Late Quaternary vegetation and climate of the Sonoran Desert, United States and Mexico. In: Betancourt, J. L., T. R. Van Devender y P. S. Martin (eds.). Packrat Middens.The Last 40,000 years of Biotic Change. The University of Arizona Press. Tucson, Arizona. pp. 134-164.

Van Devender, T. R., R. Thompson y J. L. Betancourt. 1987. Vegetation history of the deserts of southwestern North America; The nature and timing of the Late WisconsinHolocene transition. In: Ruddiman, W. F. y H. E. Wright Jr. (eds.). North America and adjacent oceans during the last deglaciation. Geological Society of America. Boulder, Colorado. pp. 323-352.

Van Devender, T. R., A. L. Reina G., M. C. Peñalba y C. I. Ortega-Rosas. 2003. The Ciénega de Camilo: a threatened habitat in the Sierra Madre Occidental of eastern Sonora, Mexico. Madroño 50: 187-195.

Van Devender, T. R. y A. L. Reina G. 2005. Floristic comparison of an Arizona 'sky island' and the Sierra Madre Occidental in eastern Sonora: the Huachuca Mountains and the Yécora area. In: Gottfried, G. J., B. S. Gebow, L. G. Eskew y C. B. Edminster (comps.). Connecting mountain islands and desert seas: biodiversity and management of the Madrean Archipielago II. Proc. of the Madrean Archipelago. United States Departmente of Agriculture, Forest Service Proceedings RMRS-P-36. Tucson, Arizona. pp. 154-157.

Van Devender, T. R., J. R. Reeder, C. G. Reeder y A. L. Reina G. 2005. Distribution and diversity of grasses in the Yécora region of the Sierra Madre Occidental of eastern Sonora, Mexico. In: Cartron, J. L. E., G. Ceballos y R. S. Felger (eds.). Biodiversity, ecosystems, and conservation in northern Mexico. Oxford University Press. Oxford. pp. 107-121.

Van Geel, B. 1978. A paleoecological study of Holocene peat bog sections in Germany and The Netherlands. Rev. Palaeobot. Palynol. 25: 1-120.

Van Geel, B., L. R. Mur, M. Ralska-Jasiewiczowa y T. Goslar. 1994. Fossil akinetes of Aphanizomenon and Anabaena as indicators for medieval phosphate-eutrophication of Lake Gosciaz (Central Poland). Rev. Palaeobot. Palynol. 83: 97-105.

Van Geel, B., V. Odgaard y M. Ralska-Jasiewiczowa. 1996. Cyanobacteria as indicators of phosfate-eutrophication of lakes and pools in the past. PACT 50(1): 399-415.

Wright, H. E. Jr. 1991. Coring tips. J. Paleolimnol. 6(1): 37-49. 